\title{
Diffuse cerebrospinal gliomatosis presenting as motor neuron disease for two years
}

\author{
MANFRED SCHMIDBAUER, ${ }^{*}$ CHRISTIAN MÜLLER, $†$ IVO PODREKA, $\dagger$ \\ BRUNO MAMOLI, $\ddagger$ ELFRIEDE SLUGA,§ LÜDER DEECKE† \\ From the Neurological Institute, ${ }^{*}$ the Neurological Clinic, $\dagger$ Kaiser Franz Josef Hospital $\ddagger$ and Wilhelminen \\ Hospital, Vienna, Austria
}

SUMMARY A patient with symptoms and signs of motor neuron disease for 2 years finally developed sensory disturbances and increased intracranial pressure. MRI and CT showed enlargement of the right side of the cerebellum, the brainstem and parts of the cerebral hemisphere with focal hyperperfusion demonstrated by SPECT. Necropsy revealed a diffuse cerebrospinal gliomatosis with loss of spinal motor neurons in tumour infiltration of the anterior horns. This type of spinal cord involvement is considered responsible for the unusual clinical presentation of the neoplasm.

Diffuse gliomatosis is a very rare entity among neuroepithelial neoplasms which involves the whole brain or large parts of it. ${ }^{1}$ A characteristic presentation of neurological deficits is not found. Signs of increased intracranial pressure, behavioural and mental changes, seizures and focal neurological impairments are inconstantly present. ${ }^{2}$ Diagnostic problems also arise from non-specific results of radiological and laboratory investigations; CT may fail to confirm the diffuse enlargement of the brain. Hypervascularisation is usually restricted to areas of highly cellular malignant tumour, so angiography might be inconclusive. Outgrowth of tumour cells into the CSF compartments is not constant, so CSF investigations are of limited value. ${ }^{3}$ Biopsy in many cases is necessary to establish a diagnosis.

We present a clinico-pathological study of a patient with a highly unusual course in whom diffuse gliomatosis was not diagnosed until necropsy.

\section{Case report}

In June 1983, the 32 year old male patient was first admitted to the hospital with weakness of the lower limbs which had slowly progressed during the previous 6 months; there was

Address for reprint requests: Dr Manfred Schmidbauer, Neurological Institute, University of Vienna, A-1090 Vienna, Austria.

Received 14 June 1988 and in revised form 7 September 1988. Accepted 12 September 1988 also slight weakness of the upper limbs. Muscle atrophy was pronounced on the left side. The triceps surae muscles and the dorsal flexors of the feet were markedly affected resulting in a stepping gait. Fasciculation was occasionally seen and remained restricted to the wasted muscles. Both ankle jerks were diminished; however, both knee jerks were exaggerated; the plantar response was extensor on the right side and equivocal on the left. The abdominal reflexes were abolished. There was slight weakness in distal flexor functions of the upper extremities without any further abnormalities. All sensory modalities were normal and therefore no sensory nerve electrical testing was performed. All cranial nerves were normal. In July 1983 EMG recordings were performed on the left tibialis anterior and biceps brachii muscles. During voluntary movement potentials with abnormally high amplitudes were found. There was an increase of the mean potential duration up to $21.7 \mathrm{~ms}$ in the tibialis anterior muscle (normal value $13.2 \mathrm{~ms}$ ) and up to $15.2 \mathrm{~ms}$ in the biceps brachii muscle (normal value $10.2 \mathrm{~ms}$ ). The proportion of polyphasic potentials was $20 \%$ in the tibialis anterior muscle and $10 \%$ in the biceps brachii muscle. A muscle biopsy specimen was suggestive of neurogenic atrophy of the anterior horn cell type. A diagnosis of amyotrophic lateral sclerosis was made. Subsequent out-patient follow up showed slight progression of motor dysfunction. The patient was able to finish his law studies. In October 1984, sensory loss first affected the right side of the face. In September 1985, atrophy of the right masseter muscle was found. The right corneal reflex was weakened. There was binocular rotating nystagmus in upward gaze. Palsy of the right facial and hypoglossal nerves was found. Speech was dyarthric and regurgitation occurred. Tetraparesis was pronounced on the left side, tendon reflexes were exaggerated except for absent ankle jerks, Trömmner's 


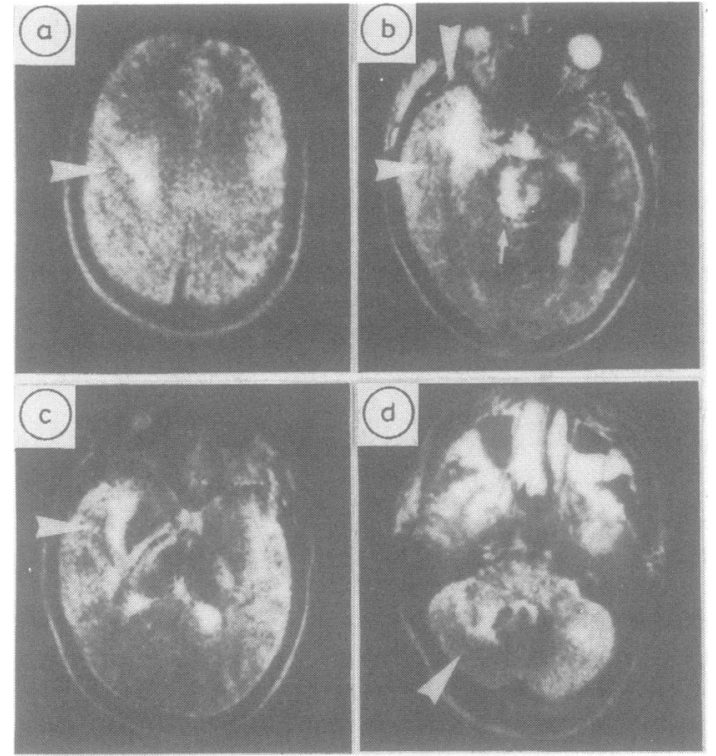

Fig $1 T 2$ weighted MRI shows high intensity signal lesions in the white matter of the right cerebral hemisphere (arrowhead a), rostral parts of the right temporal lobe (arrowheads $b$ ), the pons with accentuation on the right side (arrow b), the right caudate nucleus (arrowhead $c$ ) and the right cerebellar hemisphere (arrowhead d)

and Babinski's signs were found. EEG showed delta waves over the frontal lobe, accentuated on the left side. CSF cell count and protein content were normal. At the end of September 1985, MRI suggested diffuse increase of the white matter in the right cerebellar hemisphere and temporal lobe as well as of the whole pons in T2 weighted imaging (fig $1 \mathrm{a}-\mathrm{d})$. In SPECT these lesions showed increased uptake of N-isopropyl-(I-123)-p-iodoamphetamine (IMP). CT revealed a hypodense area in the right cerebellar hemisphere and in the ponto-mesencephalic junction. At this time the patient had left hemiataxia and diffuse headache. Mental functions deteriorated progressively, and at the end of November the patient was in a precomatous state associated with severe tetraparesis which was pronounced on the left side. CT showed a large hypodense area extending from the right parietal to the right temporal lobe leading to displacement and compression of the ventricular system. An additional hypodense lesion was found in the right cerebellum. Bulbar symptoms increased. At the end of November 1985, a biopsy specimen was taken from the right temporal lobe. Two days later the patient expired from increased intracranial pressure.

A complete necropsy showed blood aspiration from an acute gastric ulcer and severe pulmonary oedema.

\section{Neuropathological investigation}

Muscle biopsy ( 2 years and 6 months prior to death): fibre atrophy was restricted to small groups of some $4-6$ fibres (fig $2 \mathrm{~d})$. Some target fibres and internal nuclei were seen.

Brain biopsy from the right temporal lobe (two days prior to death): there was diffuse tumour infiltration of gray and white matter by densely packed cells with small rounded hyperchromatic nuclei. A perivascular and perineuronal arrangement of these cells was conspicuous (fig 2b). Immunocytochemistry for leucocyte common antigen (CLA), for glial fibrillary acidic protein (GFAP), S-100 protein and neuron specific enolase (NSE) did not reveal labelled tumour cells.

\section{Necropsy}

Brain, spinal cord and one sciatic nerve were examined. A superficial postoperative lesion was grossly visible in the right temporal lobe. Diffuse enlargement of the right cerebral and cerebellar hemispheres was evident; the midline structures were slightly displaced to the left. The brainstem was symmetrically enlarged. Spinal cord and sciatic nerve were grossly normal.

Histologically, the neoplastic infiltration affected both cerebral and cerebellar hemispheres, but was accentuated on the right side. Neoplastic cells were generally aligned along the major fibre tracts of longitudinal and transversal pathways but the major brainstem structures and proportions were preserved. The dense infiltration consisted of highly anaplastic astrocytic cells (fig 2 a). Their processes were elongated and frequently stained positive for GFAP and S100 protein.

The spinal cord was infiltrated by cells with rounded or elongated nuclei which were often indistinguishable from normal glial nuclei; more numerous cells were hyperchromatic, irregular and arranged in clusters or around nerved cells. They constantly were much more dense in the gray than in the white matter (fig $2 \mathrm{c}$ ). The spinal nerve roots showedo glial bundles. Expression of GFAP was prominent in many tumour cells whereas others were negative. The sciatic nerven appeared morphologically intact.

\section{Discussion}

To our knowledge, the reported case is unique in its clinical presentation. The neuropathological characteristics are in agreement with criteria for diagnosis of a diffuse gliomatosis as defined by Scheinker and Evens $1943,{ }^{4}$ and are also in accordance with previously described cases. ${ }^{5}$ However, the extension of the neoplastic infiltration along the entire neuraxis is exceptional. ${ }^{6-9}$

The uncommon course of the disease elicited severe diagnostic problems. Initial symptoms and signs, EMG recordings, and a muscle biopsy indicated an affection of spinal motor neurons. Therefore amyotrophic lateral sclerosis was diagnosed. The later appearance of cerebellar symptoms and the affection of the trigeminal and facial nerves with a latency of 16 month led to diagnostic confusion. An atypical form of multiple sclerosis or encephalitis with involvement of the peripheral nerve roots or even Lyme disease (evaluation of specific IgG and IgM in paired CSF and serum probes were not diagnostic) were considered as possible cause for the clinical presentation. During the final course, when MRI, CT and SPECT demonstrated lesions, a diagnosis of a hyperfused (possibly 


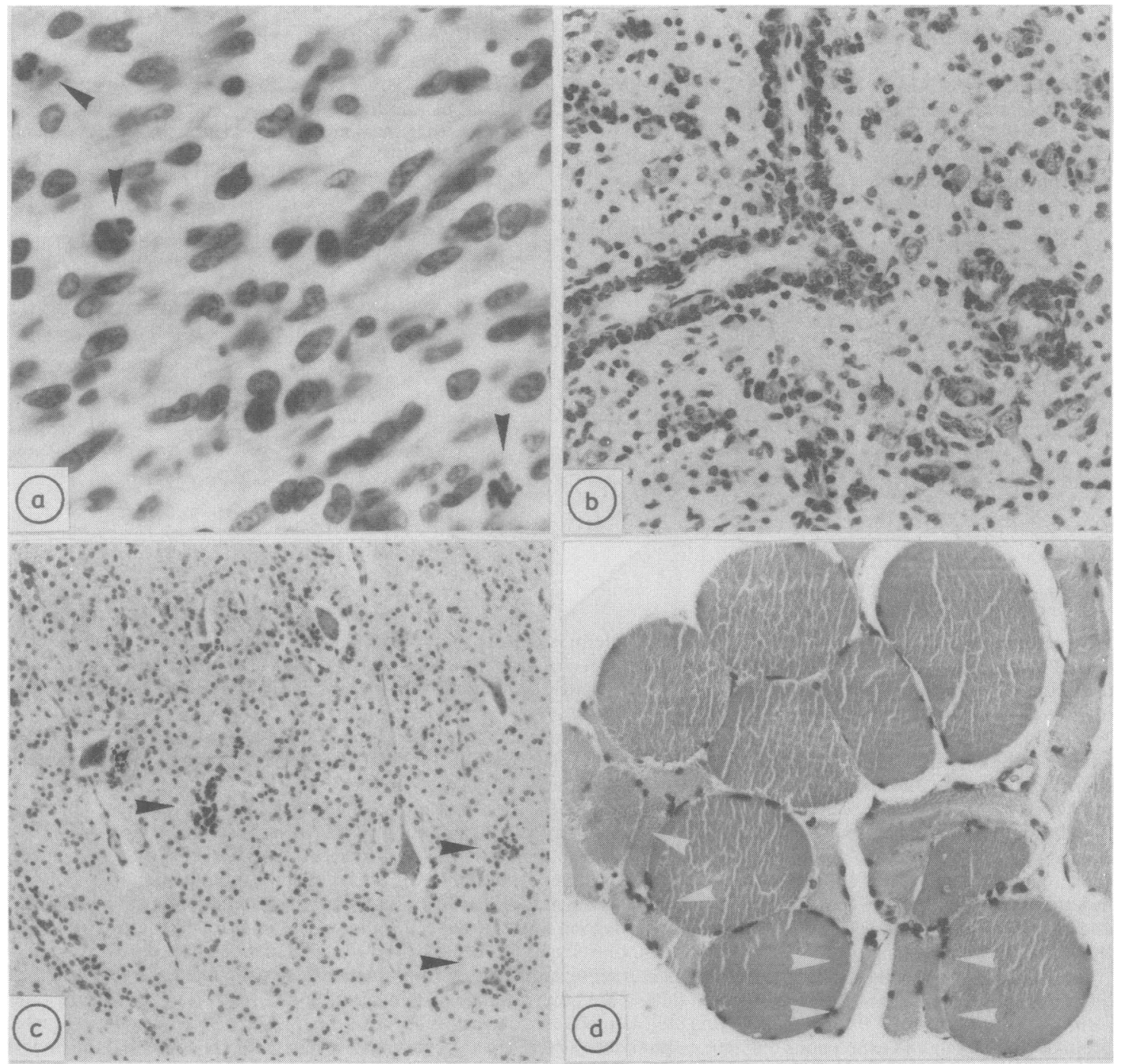

Fig 2 Histopathology of the mesencephalic level at necropsy (a) shows densely packed irregular hyperchromatic tumour cell nuclei and frequent mitoses (arrowheads). The biopsy specimen shows the perivascular and perineuronal arrangement of undifferentiated tumour cells $(b)$. In the spinal cord at necropsy large motor neurons of the anterior horn are frequently surrounded by densely packed tumour cell nuclei (arrowheads) whereas others remain unaffected (c). In the muscle biopsy specimen small groups of atrophied fibres can be seen (d). $a$ and $d: H$ and $E, \times 720, b: H$ and $E, \times 282, c: H$ and $E, \times 180$.

hypermetabolic?) multifocal malignant brain tumour casually associated with amyotrophic lateral sclerosis was made. It remains obscure why spinal motor areas were predominantly affected in early stages of the disease. In similar clinical courses, the combination of signal intense areas seen on MRI scans associated with hyperperfusion in SPECT or PET images might lead to a correct diagnosis and permit earlier performance of brain biopsy. Appropriate early antineoplastic therapy is not feasible as long as cerebrospinal gliomatosis mimicks a variety of non-neoplastic conditions.

\section{References}

1 Zülch KJ. Histological typing of tumors of the central nervous system. Geneva: World Health Organisation, 1979.

2 Grant N. Diffuse glioblastosis. In: Vinken PJ, Bruyn GW, 
eds. Handbook of clinical neurology vol 18. Amsterdam: North Holland Publishing Company, 1975: 73-9.

3 Wechsler LR, Gross RA, Miller DC. Meningeal gliomatosis with "negative" CSF cytology: the value of GFAP staining. Neurology 1984;34:1611-5.

4 Scheinker IM, Evans JP. Diffuse cerebral glioblastosis. $J$ Neuropathol Exp Neurol 1943;2:78-189.

5 Artigas J, Cervos-Navarro J, Iglesias JR, Ebhardt G. Gliomatosis cerebri: clinical and histological findings. Review article. Clin Neuropathol 1985;4:135-48.
6 Kawano N, Miyasaka Y, Yada K, Atari H, Sasaki K. Diffuse cerebrospinal gliomatosis: case report. $J$ Neurosurg 1978;49:303-7.

7 Ferraro A, Jervis GA, Sherwood WD. Patchy blastomatous infiltration of the central nervous system (patchy schwannosis?). J Neuropathol Exp Neurol 1943;2:207-25.

8 Moore MT. Diffuse cerebrospinal gliomatosis, masked by syphilis. J Neuropathol Exp Neurol 1954;13 129-43.

9 Nevin S. Gliomatosis cerebri. Brain 1938;61:170-91.

\section{Bromide: from neurology to English}

When a neurologist speaks of a "clinical bromide", the term is most fitting; indeed, the neurologist is entitled to use it in ano $\mathrm{N}$ almost proprietary manner. The derogatory sense of the word came into usage years after bromides were first given in the $\mathrm{O}$ treatment of neurological (particularly epilepsy) and psychiatric disorders. The word bromide can be traced from its chemical origin to its second meaning in literate English usage via neurology.

Bromine was discovered in 1826 by a French chemist, Antoine Balard, and was subsequently named such after the Gree bromos ("stink"). It seems that Balard called it muride, a name which was later changed to bromine by the Academie Francaise;' however, the naming of bromine is attributed to Balard elsewhere. ${ }^{2}$

Though others may have been instrumental in propagating the use of bromides for epilepsy, ${ }^{3}$ Sir Charles Locock has bee given priority. He commented on his success with potassium bromide at a meeting in 1857, after Sieveking had spoken on the current state of therapeutics. ${ }^{4}$ Locock was not a neurologist, but an obstetrician, and accoucheur to the high-born, including Queen Victoria. Locock had apparently read a case report of impotence following potassium bromide ingestion. His Victorian notions on the origins of epilepsy (as stemming from onanism in some cases) led him to try the bromide as an antiaphrodisiac, and therefore antiepileptic, drug; he had first used it with good results in women with hysteria, and later, hysterical epilepsy. ${ }^{56}$ It became the mainstay in the treatment of seizures until well into this century, and was widely used as a sedative as well.

Priority for the use of bromide in the nonelemental sense is open to question. According to the Dictionary of American Slang, Gelett Burgess coined the slang (for that time, if not now) meaning, with his 1906 book, Are You a Bromide?' Another source gives this account: "Bromo-seltzer being used as a sedative, the magazine Smart Set, in April, 1906, suggested the word bromide for persons and expressions that tend to put one to sleep." ${ }^{8}$ In any event, it is fortunate for epileptics that the English usage is now more common than the neurological.

Other word meanings have been introduced into English via neurologic disorders. Most are generally regarded as slang, including "had a fit", "hysterical", and perhaps the youthful usages of "hyper" and "spastic". "Knee jerk" is another of these slang terms, used mainly as a modifier before the contrary of one's politics. The term in the neurological sense was first used by W R Gowers in 1879; the reflex had been described under other names before. ${ }^{9}$

K G WOODWARD, MD, The Hammond Clinic, 7905 Calumet, Munster, IN, USA 46321.

\section{References}

1 Academic American Encyclopedia. Danbury, CN: Grolier Inc., 1985:502.

2 Klein E. A Comprehensive Etymological Dictionary of the English Language. Amsterdam: Elsevier, 1966:202.

3 Friedlander WJ. Who was "the father of bromide treatment of epilepsy"? Arch Neurol 1986;43:505-7.

4 Locock C, in discussion, Sieveking EH. Analysis of 52 cases of epilepsy observed by the author. Lancet 1857;i:527-8.

5 Joynt RJ. The use of bromides for epilepsy. AJDC 1974;128:362-3.

6 Lennox WG. The centenary of bromides. $N$ Engl J Med 1957;256:887-90.

7 Wentworth H, Flexner SB, eds. Dictionary of American Slang, Second Supplemental Edition. New York: Thomas Y. Crowell Co., 1975:64.

8 Shipley JT. Dictionary of Word Origins, 2nd ed. New York: Greenwood Press, 1945:58.

9 Tyler KL, McHenry LC. Fragments of neurological history[:] The knee jerk and other tendon reflexes. Neurology 1983;33:609-10. 